\title{
Quantification de l'érosion hydrique, par la méthode RUSLE, au niveau du bassin versant de l'Oued Mikkès en amont du barrage Sidi Chahed (région de Meknès, Maroc)
}

\author{
Kaouthar Mazouzi \\ Abdellah El Hmaidi
}

Université Moulay Ismail, Faculté des Sciences, Département de Géologie, Equipe Sciences de l'Eau et Ingénierie de l'Environnement, Zitoune,

Meknès, Maroc

Rachid Bouabid

Ecole nationale d'Agriculture de Meknès, Meknès, Maroc

El Mâti El Faleh

Université Moulay Ismail, Faculté des Sciences, Département de Géologie, Equipe Sciences de l'Eau et Ingénierie de l'Environnement, Zitoune,

Meknès, Maroc

Doi:10.19044/esj.2021.v17n14p256

Submitted: 10 March 2020

Accepted: 13 April 2021

Published: 30 April 2021
Copyright 2021 Author(s)

Under Creative Commons BY-NC-ND

4.0 OPEN ACCESS

Cite As:

Mazouzi K., El-Hmaidi A., Bouabid R. \& El-Faleh E-M. (2021). Quantification de l'érosion hydrique, par la méthode RUSLE, au niveau du bassin versant de l'Oued Mikkès en amont du barrage Sidi Chahed (région de Meknès, Maroc). European Scientific Journal, ESJ, 17(14), 256. https://doi.org/10.19044/esj.2021.v17n14p256

\section{Resume}

La présente étude a pour but d'estimer les pertes en sol dans le bassin versant de l'Oued Mikkès en amont du barrage Sidi Chahed situé dans la région de Meknès (Maroc), par l'utilisation de l'équation universelle révisée des pertes en sol de (Wischmeier et Smith, 1978). La spatialisation du modèle empirique s'est basée sur l'intégration des cinq facteurs dans le Système d'Information Géographique (SIG), à savoir, l'érosivité des pluies « R », l'érodibilité des sols « $\mathrm{K} »$, le couvert végétal « $\mathrm{C} »$, la topographie « $\mathrm{LS}$ » et les pratiques antiérosives « $\mathrm{P} »$, et dont la superposition a permis d'élaborer la carte quantitative des pertes en sols à l'échelle du bassin versant. Le bassin versant de l'Oued Mikkès s'étale sur une superficie de $1444 \mathrm{~km}^{2}$; il est de forme allongée avec une altitude allant de $2090 \mathrm{~m}$ à $184 \mathrm{~m}$ du Sud vers le 
Nord et une pente qui varie de 0 à $189 \%$. L'étude pluviométrique des onze stations climatiques qui se trouvent au niveau du bassin versant a démontré l'irrégularité des précipitations dans le temps et dans l'espace. Le facteur d'érosivité R a présenté des valeurs allant de 60 à 119, avec une moyenne de $85 \mathrm{Mj} . \mathrm{mm} / \mathrm{ha}$. h. an (Millijoule. Millimètres/hectare. Heure. An). Le facteur d'érodibilité $K$ présente des valeurs de 0 jusqu'à 0,6 et il a montré que les sols peu évolués et les vertisols sont les plus vulnérables à l'érosion. Le facteur LS relatif à la topographie varie entre 0 et 2562, dont l'amont est plus accidenté et plus sensible. Le facteur $\mathrm{C}$ diffère selon l'occupation des sols, d'où les sols des parcours, les incultes et les sols cultivés sont les plus fragiles. L'érosion hydrique affecte toutes les parties du bassin versant mais avec des degrés variables selon les facteurs de l'érosion. Les terres en dessous du seuil de tolérance de $7 \mathrm{t} / \mathrm{ha} /$ an ne représentent que $11 \%$ de la superficie du bassin, celles présentant des pertes variant entre 7 et 20 t/ha/an occupent $53 \%$, le facteur d'érosion dans $24 \%$ de la superficie a des valeurs entre 20 et $40 \mathrm{t} / \mathrm{ha} / \mathrm{an}$ et celles dépassant les $40 \mathrm{t} / \mathrm{ha} / \mathrm{an}$ affectent environ $12 \%$ du territoire.

Mots clés : Barrage Sidi Chahed, Bassin versant, Oued Mikkès, Erosion hydrique, RUSLE

\title{
Quantification of water erosion in the catchment area of Oued Mikkes upstream of Sidi Chahed dam (Meknes region, Morocco)
}

\author{
Kaouthar Mazouzi \\ Abdellah El Hmaidi \\ Université Moulay Ismail, Faculté des Sciences, Département de Géologie, \\ Equipe Sciences de l'Eau et Ingénierie de l'Environnement, Zitoune, \\ Meknès, Maroc \\ Rachid Bouabid \\ Ecole nationale d'Agriculture de Meknès, Meknès, Maroc \\ El Mâti El Faleh \\ Université Moulay Ismail, Faculté des Sciences, Département de Géologie, \\ Equipe Sciences de l'Eau et Ingénierie de l'Environnement, Zitoune, \\ Meknès, Maroc
}

\begin{abstract}
The purpose of this study is to estimate soil losses at the Mikkes watershed upstream of the Sidi Chahed dam in the Meknes region (Morocco), using the revised universal equation of losses in soil of (Walter $\mathrm{H}$ Wischmeier
\end{abstract}


et Smith, 1978). The spatial empiric model was based on the integration of the five factors in the Geographical Information System (GIS), namely, the erosivity of rains " $R$ ", the erodibility of soils "K", the vegetation cover " $C$ ", topography "LS" and anti-erosion practices "P", the superposition of which allowed the development of a quantitative map of soil losses at the scale of the watershed. The catchment area of Oued Mikkes covers an area of $1444 \mathrm{~km} 2$; it is of elongated shape with an altitude ranging from 2090 meters to 184 meters from the South to the North, and a slope which varies from 0 to $189 \%$. The rainfall survey of the eleven climatic stations located at the catchment level has shown the irregularity of precipitation in time and space. The erosivity factor $\mathrm{R}$ showed values ranging from 60 to 119, with an average of $85 \mathrm{Mj} . \mathrm{mm} / \mathrm{ha}$.h.a ". Erodibility factor $\mathrm{K}$ showed values from 0 to 0.6 and showed that poor soils and vertisols are the most vulnerable to erosion. The LS topography factor varies between 0 and 2562, the upstream of which is more rugged and more sensitive. The $\mathrm{C}$ factor differs according to the land use, from which the soils of the rangelands, the uncultivated and the cultivated soils are the most fragile. Water erosion affects all parts of the watershed, but with varying degrees depending on the factors of erosion. Land below the tolerance level of $7 \mathrm{t} / \mathrm{ha} /$ year represents only $11 \%$ of the catchment area, those with losses varying between 7 and $20 \mathrm{t} / \mathrm{ha} /$ year occupy 53\%, the erosion factor in $24 \%$ of the area has values between 20 and $40 \mathrm{t} /$ ha / year and those exceeding $40 \mathrm{t} / \mathrm{ha} /$ year affect about $12 \%$ of the territory.

Keywords: Sidi Chahed Dam, Watershed, Mikkes wadi, Water Erosion, RUSLE

\section{Introduction}

L'érosion hydrique est un processus qui s'exprime par la dégradation des sols. Elle menace une grande surface à l'échelle du globe et décrit les phénomènes dus à l'homme et/ou à l'agressivité climatique qui réduit le potentiel de production des sols et la qualité des ressources naturelles. Elle constitue la première manifestation du phénomène de la désertification et se focalise surtout dans les régions à climat semi- aride et dans les zones méditerranéennes.

L'érosion hydrique se considère comme un phénomène important influant sur l'environnement. Elle a des conséquences négatives sur le cadre socio-économique à l'échelle locale, régionale et nationale, d'une manière générale, à cause de la croissance démographique et aussi des changements climatiques. Elle constitue un danger sur les infrastructures, sur les productions agricoles et aussi sur la qualité des eaux.

Une étude concernant la dégradation des sols au Maroc, faite par la l'Organisation des Nations Unies pour l'Alimentation et l'Agriculture (FAO), 
a montré l'ampleur de ce phénomène avec une valeur de 12,6 millions d'hectares de culture et de parcours qui ont été considérés en menaces (Zouagui et al., 1977). Une autre étude plus récente de la FAO, en 1990, a trouvé qu'un pourcentage de $40 \%$ des terres de l'ensemble du territoire était affecté par l'érosion hydrique (Lufafa et al., 2003).

La dégradation spécifique moyenne dépasse les $50 \mathrm{~T} / \mathrm{ha} / \mathrm{ha}$ au niveau de plusieurs zones, notamment au Rif à cause de l'agressivité climatique, du relief accidenté, de la grande sensibilité des couches lithologiques et de l'impact du facteur anthropique (Ait Fora, 1995; Lahlou, 1977). Ceci se traduit par une dégradation importante et inquiétante de la fertilité et de la productivité des sols. Mais également par la réduction de la capacité du stockage des eaux par l'effet de l'envasement des barrages.

Les plus grands barrages du Maroc recevraient chaque année environ 50 millions de tonnes de sédiments (Marzouki, 1992). Le taux de comblement varie de $0,1 \mathrm{~mm} 3 / \mathrm{an}$ au niveau du barrage $\mathrm{Smir}$ à $10 \mathrm{~mm} 3 / \mathrm{an}$ au niveau du barrage Mohammed V.

Le présent travail a pour objectif l'évaluation de l'ampleur du risque d'érosion dans le bassin versant de l'Oued Mikkès en amont du barrage Sidi Chahed ainsi que la réalisation du modèle spatial des pertes en sol à l'aide de la télédétection et du système d'information géographique (SIG). Par ailleurs, il existe plusieurs modèles pour la quantification de l'érosion hydrique et le choix se fait en fonction des données disponibles. La méthode la plus utilisée à l'échelle nationale est l'équation universelle révisée des pertes en sol (USLE) de (Wischmeier and Smith, 1978). Elle permet de calculer et d'analyser les différents facteurs qui influencent sur l'érosion tels que le facteur d'érosivité des pluies, le facteur d'érodibilité des sols, la topographie, le couvert végétal et les pratiques culturales.

\section{Materiel et Methodes}

\subsection{Présentation de la zone d'étude}

Le bassin versant de l'Oued Mikkès est situé à l'amont du barrage Sidi Chahed qui fait son départ aux bordures des montagnes du Moyen Atlas au Sud et traverse la plaine du Saïs du Sud vers le Nord à presque mi-chemin des villes de Meknès et Fès. Il se termine au niveau d'un exutoire où a été édifié le barrage Sidi Chahed qui a comme coordonnées (ABHS, Fès): $X=507200 \mathrm{~m}$ $\mathrm{Y}=389500 \mathrm{~m}$, ayant une capacité de $170 \mathrm{~mm} 3$ qui contribuent à la régularisation des eaux du grand bassin versant de l'Oued Sebou (Figure 1): 


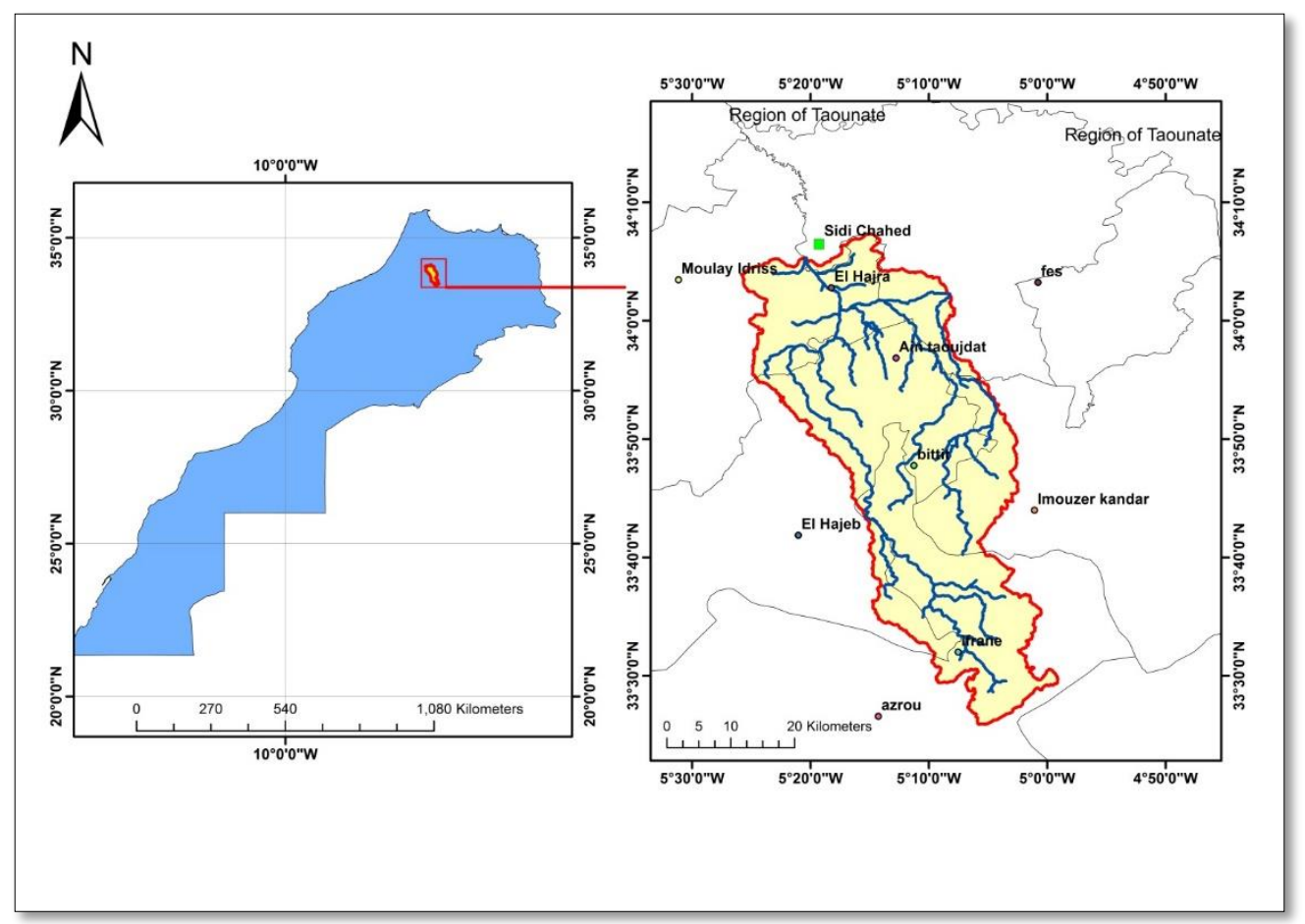

Figure 1 : Situation géographique, réseau hydrographique et découpage du bassin versant de l'Oued Mikkès.

\subsection{Méthodes}

\subsubsection{Taux annuel de perte en sol}

La quantification des pertes en sol au niveau du bassin versant de l'Oued Mikkès a été réalisée en adoptant l'Equation Universelle Révisée des Perte en Sol (RUSLE):

\section{$A=$ R. K. LS. C. P}

Avec :

A : Taux annuel de perte en sol en $\mathrm{t} / \mathrm{ha} / \mathrm{an}$;

$\mathrm{R}$ : Facteur d'érosivité des pluies;

$\mathrm{K}$ : Facteur d'érodibilité des sols;

LS : Facteur de topographie (Longueur et inclinaison de la pente);

$\mathrm{C}:$ Facteur de couverture végétale;

$\mathrm{P}:$ Facteur des pratiques culturales antiérosives.

\subsubsection{Le facteur d'érosivité des pluies « $R$ »}

L'absence des données de l'énergie cinétique (Ec) et l'intensité moyenne des précipitations en $30 \mathrm{~mm}$ empêche l'utilisation de la formule de Wischmeier. Dans ce cas, le facteur d'érosivité des pluies R a été estimé par la formule proposée par Rango et Arnoldus (1987) qui exige l'utilisation des 
données météorologiques sous forme des moyennes mensuelles et annuelles et dont la formule est la suivante :

\section{$\log \mathbf{R}=1,74 . \log \Sigma\left(\mathrm{Pi}^{2} / \mathrm{P}\right)+1,29$}

Avec:

$\mathrm{R}$ : Agressivité pluviale;

$\mathrm{Pi}$ : Moyenne des précipitations mensuelles en mm;

$\mathrm{P}$ : Moyenne des précipitations annuelles en $\mathrm{mm}$.

Cette formule a été appliquée sur les données des pluviométries enregistrées par onze stations climatiques situées à l'intérieur ou à proximité du bassin versant (Figure 2).

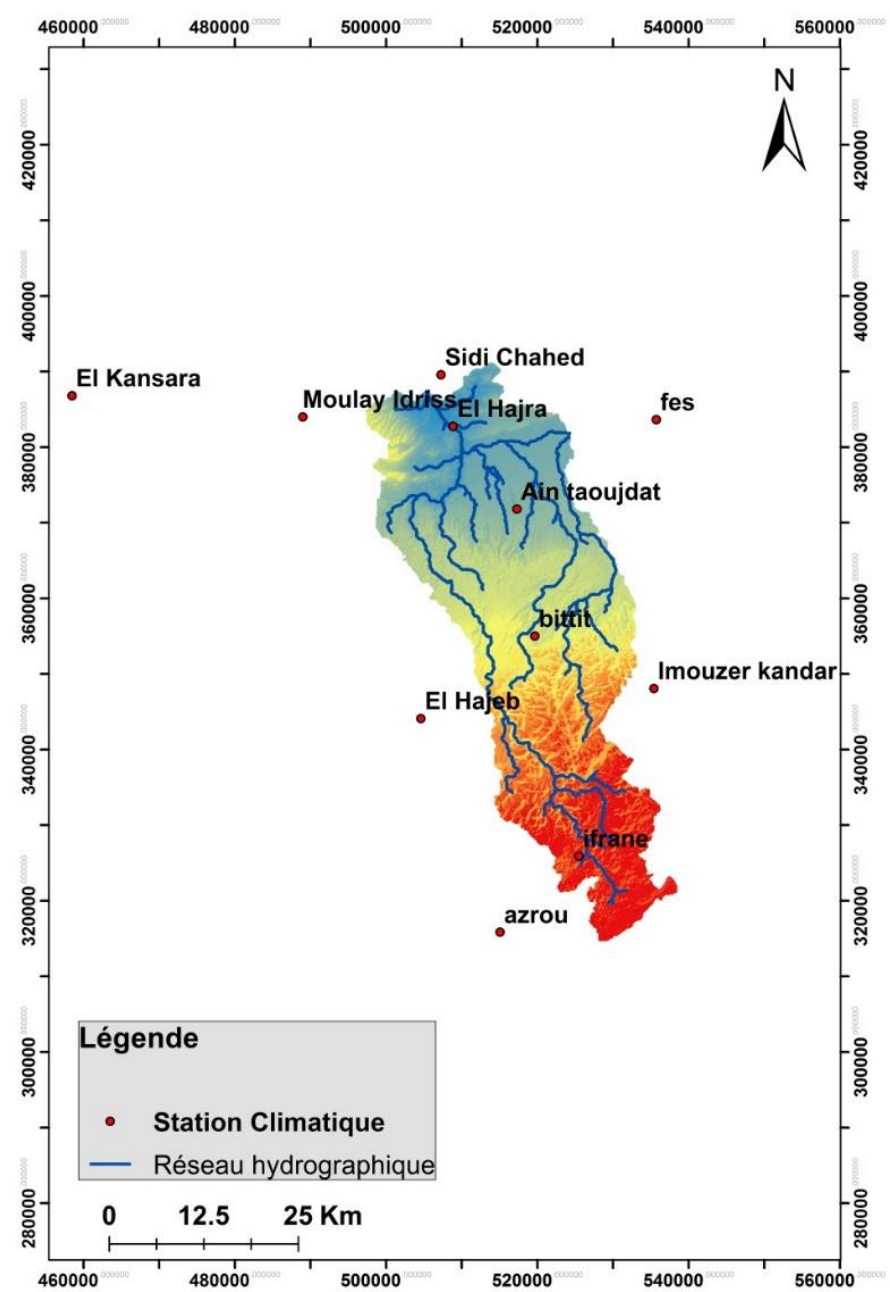

Figure 2 : Carte de situation des stations climatiques. 


\subsubsection{Le facteur d'érodibilité des sols « $K$ »}

L'érodibilité des sols dépend du pourcentage de matière organique, de la texture, de la structure et de la perméabilité du sol. Ce facteur est défini comme étant la capacité d'un sol à résister à l'érosion, il s'exprime en t.ha.H/ha.Mj.mm. Son estimation a été faite à l'aide l'équation de Wischmeier et Smith (1978) suivante :

$$
100 \mathrm{~K}=2,1 . \mathrm{M}^{1,14} \cdot 10^{-4}(12-a)+3,25(\mathrm{~b}-2)+2,5(c-3)
$$

Avec:

$\mathrm{K}$ : Facteur d'érodibilité en t.ha.H/ha.Mj.mm (Tonne. Hectare. Heure/Hectare. Mégajoule. Millimètre);

$\mathrm{M}$ : Représente la texture et se calcul par la formule ;

$\mathrm{M}=(\%$ sable fin + limon $) *(100-\%$ argile $) ;$

$\mathrm{a}:$ Pourcentage de matière organique;

$\mathrm{b}$ : Code des classes de structure ;

c : Code des classes de perméabilité.

Les paramètres pour déterminer le facteur d'érodibilité $\mathrm{R}$ ont été obtenus à partir des études pédologiques chevauchant sur le bassin versant. Les données pédologiques ont été recueillies à partir de différentes études réalisées sur les régions de Fès, Sefrou, Meknès et El Hajeb. Les paramètres $b$ et $\mathrm{c}$ ont été définis d'après la base des données de la classe structurale et de la texture du sol de la zone d'étude.

\subsubsection{Le facteur topographique « $L S$ »}

Le facteur topographique LS (sans dimension), se détermine par la longueur de la pente $\mathrm{L}$ en $(\mathrm{m})$ et par le degré d'inclinaison S (\%). Il représente l'effet de la géomorphologie sur l'érosion hydrique. Pour estimer ce facteur la formule de (Moore and Wilson, 1992) a été adoptée :

$$
L S=(m+1) \cdot\left[\frac{A}{22.1}\right]^{m} \cdot\left[\frac{\sin (\beta)}{0.09}\right]^{n}
$$

Avec :

A : la surface en $\mathrm{Km} 2$ à l'amont contribuant à l'accumulation du flux en un point donné ;

B : la pente exprimée en radians ;

Les paramètres $\mathrm{m}$ et $\mathrm{n}$ sont des constantes dont les valeurs varient comme suit : $\mathrm{m}=0,2$ à 0,6 et $\mathrm{n}=1$ à 1,3 . 
Les faibles valeurs sont utilisées quand il s'agit d'une dominance d'érosion en nappe, et les valeurs maximales sont utilisées quand il y a dominance d'une érosion en rigole. En raison de l'absence d'information détaillée sur la forme dominante de l'érosion, les valeurs de $\mathrm{m}=0,5$ et $\mathrm{n}=1,3$ ont été adoptées. Cette formule est traduite sous SIG en utilisant la formule suivante :

$$
\text { LS } \left.=\left[(\text { AccFlux. Dimpixel } / 22.1)^{0,5} \cdot\left(\text { Sin. }\left(\text { Pente }{ }_{\text {deg. }} \cdot 3.14 / 180\right) / 0.09\right)^{1.3}\right)\right] .1 .3
$$

Avec :

AccFlux : carte d'accumulation des flux;

Dimpixel : dimension des pixels de la carte d'accumulation des flux; Pentedeg : carte des pentes exprimée en degré;

$(3,14 / 180)$ : facteur de conversion des degrés en radians.

\subsubsection{Le facteur du couvert végétal « $C$ »}

Le facteur C (sans dimension) repose sur la densité et la hauteur de la couverture végétale de la surface du sol (Wischmeier et Smith 1978). Il se base sur la hauteur de la végétation, l'indice de recouvrement, le pourcentage de la couverture herbacée et les résidus de végétation ou litière.

Les valeurs de $\mathrm{C}$ varient entre 0.001 pour un terrain entièrement couvert et 1 pour un terrain de jachère (Wischmeier and Smith, 1978).

Pour la présente étude, le couvert végétal a été obtenu à partir de la classification supervisée de l'extrait de l'image satellitaire LANDSAT-8 captée en Mars 2014. Les valeurs attribuées aux différents types de couvert végétal sont présentés dans le tableau 1 ci-dessous.

Tableau 1 : Types de couvert végétal et valeurs de C.

\begin{tabular}{|c|c|}
\hline Type du couvert végétal & Facteur C \\
\hline Forêt dense & 0,1 \\
\hline Forêt moyennement dense & 0,25 \\
\hline Forêt claire & 0,4 \\
\hline Terrain de parcours & 0,7 \\
\hline Terrain de cultures ou jachères & 0,5 \\
\hline Terrain de cultures plus ou moins dégradés & 0,8 \\
\hline Terrain incultes, zones urbaines et routes & 1 \\
\hline Surface d'eau & 0 \\
\hline
\end{tabular}

\subsubsection{Le facteur des pratiques anti-érosives « $P$ »}

Le facteur $P$ traduit l'influence des pratiques culturales qui permettent de réduire la quantité et la vitesse des eaux ce qui abaisse le degré de ruissellement et par conséquent le degré de l'érosion.

Vu que les données sur les aménagements antiérosifs ne sont pas disponibles, la valeur de 1 a été attribuée au facteur P sur toute la surface du bassin versant. 


\section{Resultats et Discussion}

\subsection{Le facteur d'érosivité « $R$ »}

L'étude pluviométrique des onze stations climatiques a montré une irrégularité des précipitations dans le temps et dans l'espace, avec des précipitations moyennes annuelles variant entre $965 \mathrm{~mm}$ pour la station d'Ifrane et $283 \mathrm{~mm}$ au niveau de la station d'EL Kansara (Tableau 2).

Tableau 2 : Moyenne des précipitations annuelles en mm et Valeurs de R.

\begin{tabular}{|c|c|c|}
\hline Station & $\begin{array}{l}\text { Précipitation moyenne } \\
\text { annuelle }(\mathrm{mm})\end{array}$ & $\mathrm{R}$ \\
\hline Ifrane & 965 & 127 \\
\hline Azrou & 723 & 99 \\
\hline Imouzzer & 608 & 87 \\
\hline El Hajeb & 575 & 84 \\
\hline Bittit & 504 & 77 \\
\hline My Idriss & 599 & 89 \\
\hline AïnTaoujdat & 448 & 71 \\
\hline El Hajra & 365 & 61 \\
\hline Sidi Chahed & 384 & 65 \\
\hline El Kansara & 283 & 56 \\
\hline Fes & 367 & 67 \\
\hline
\end{tabular}

La carte d'érosivité des pluies obtenue à partir de l'analyse spatiale des valeurs du facteur $\mathrm{R}$ calculées dans les différentes stations climatiques montre un important gradient de l'amont vers l'aval (Tableau 2), avec des valeurs allant de moins 60 à plus de 130. Le taux de précipitations est plus élevé dans les régions du Moyen Atlas à cause du relief fort (Ifrane, Azrou et Imouzer), par contre, dans la plaine de Saïs, une zone qui se caractérise par un faible relief, le taux de précipitation est moins élevé (Fès, El kansara, El Hajria). Par conséquent, le climat montagneux du Moyen Atlas a plus d'impact sur le facteur R que le climat de la plaine du Saïs et des Rides Pré-rifaines.

La répartition des classes de ce facteur en termes de superficies (Tableau 3) montre que près de 70\% du territoire sont inférieures à 90, alors que le reste du bassin versant présente des valeurs supérieures à 90 . C'est la zone amont, montagneuse, qui est la plus soumise à l'agressivité du climat (Figure 3).

Ces résultats sont comparables à ceux trouvés dans le bassin versant du haut Oum Rbia, Yjjou et al. (2014), cette étude a prouvé que $93 \%$ de la superficie du bassin est soumise à une érosivité qui varie de 70 à 119, la classe qui témoigne une érosivité modérée entre 100 et 119 occupe $24 \%$ du bassin et seulement $7 \%$ de la surface du bassin est soumise à une faible érosivité.

Aussi, dans l'étude faite par la DREF ( 2007) du NE sur le bassin versant d'Oued Beht, l'agressivité climatique obtenue est très concentré dans l'intervalle 70 à 100 avec $86 \%$. Ces résultats montrent que l'agressivité pluviométrique est forte selon la classification de Manrique (1988). 
Tableau 3. Superficie et pourcentage des classes d'érosivité pluviale R.

\begin{tabular}{|c|c|c|}
\hline Classe d'érosivité & Superficie (ha) & Superficie (\%) \\
\hline$<60$ & 1026 & 1 \\
\hline $60-70$ & 42769 & 30 \\
\hline $70-80$ & 45206 & 31 \\
\hline $80-90$ & 12723 & 9 \\
\hline $90-100$ & 7657 & 5 \\
\hline $100-110$ & 8846 & 6 \\
\hline $110-120$ & 7897 & 6 \\
\hline $120-130$ & 9178 & 6 \\
\hline$>130$ & 9186 & 6 \\
\hline \multicolumn{2}{|c|}{}
\end{tabular}

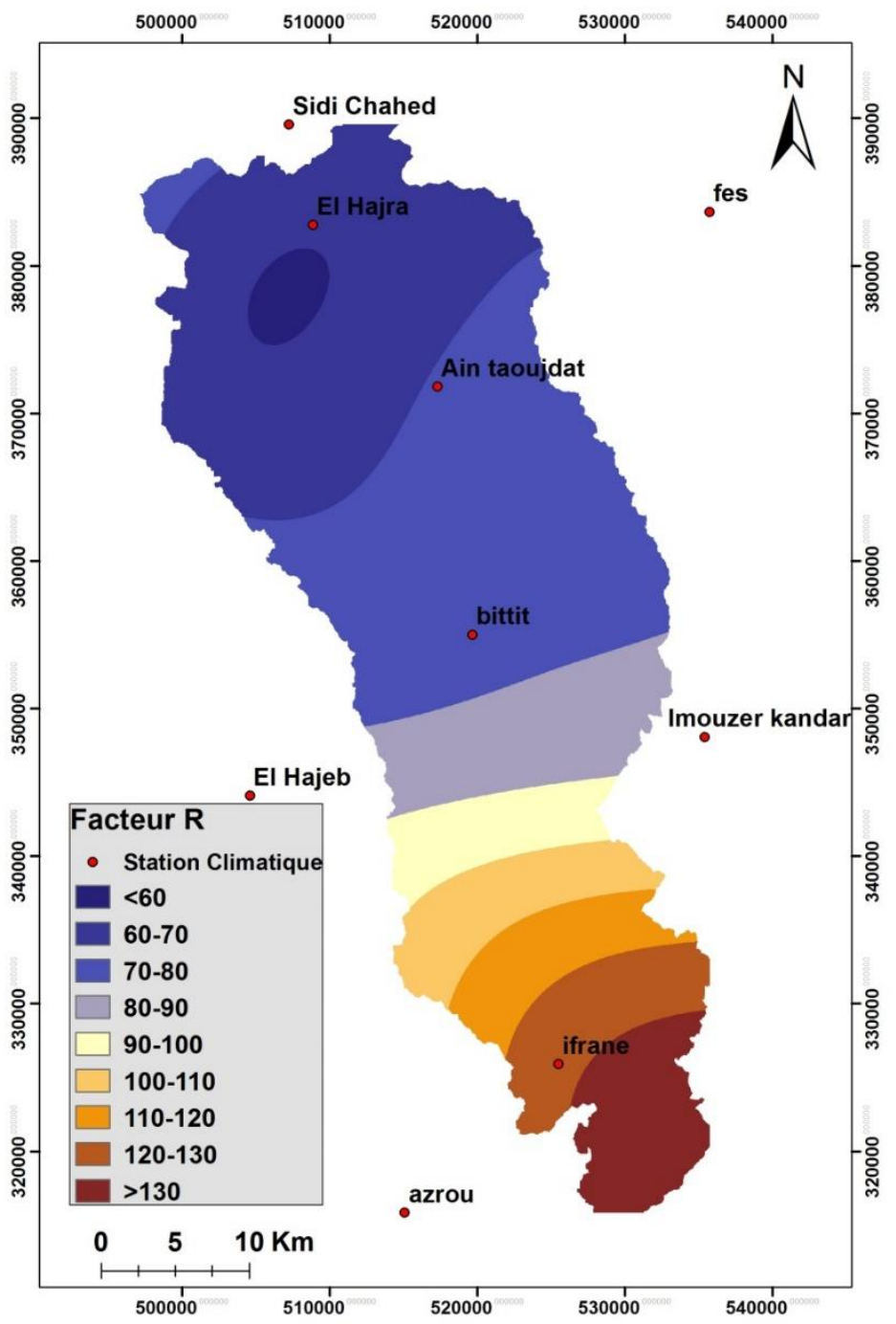

Figure 3. Carte du facteur d'érosivité R au niveau du bassin versant de l'Oued Mikkès. 


\subsection{Le facteur d'érodibilité du sol « $K$ »}

L'érodibilité du sol K varie selon le type de sol à l'échelle du bassin versant (Figure 4). Les sols peu évolués sur roches mères marneuses (régosols) ainsi que les sols à texture lourdes (vertisols) qui sont les plus vulnérables avec des facteurs K les plus élevés. Ceux à texture plus ou moins équilibrées et bien drainées sont les moins vulnérables (calcimagnésiques, isohumiques et fersiallitiques). Les résultats obtenus au Tableau 4, indiquent aussi que 37\% de la superficie du bassin versant possèdent une érodibilité très élevée $(0,3$ à $0,6), 49 \%$ de la superficie du bassin versant présentent une érodibilité moyenne $(0,2$ et 0,3$)$ et seulement $14 \%$ présentent une faible érodibilité $(0$ et $0,2)$.

Les résultats obtenus sont généralement comparables avec le bassin versant traité par Yjjou et al. (2014) sur le bassin versant du haut Oum Rbiaa, en fait, il a trouvé que plus de la moitié des sols du bassin versant, soit 59\%, possèdent une érodibilité très élevée atteignant des valeurs comprises entre 0,4 et 0,5 et seulement $22 \%$ de la surface du bassin possède une érodibilité faible inférieure à 0,2 .

Aussi, les résultats obtenus par Elbouqdaoui et al. (2006) dans une étude réalisée sur le bassin versant d'oued Srou ont montrés que les vertisols présentent une faible érodibilité $(0,1-0,2)$, alors que les sols peu évolués présentent une érodibilité élevée $(0,3-0,4)$.

Aussi, ces résultats coïncident avec ceux trouvés par EL Idrissi.R (1986) qui a rapporté que les sols peu évolués du bassin Oum Rbia sont localisés surtout sur des versants à forte pente, limitrophes des vallées où la dégradation des sols est très poussée. 


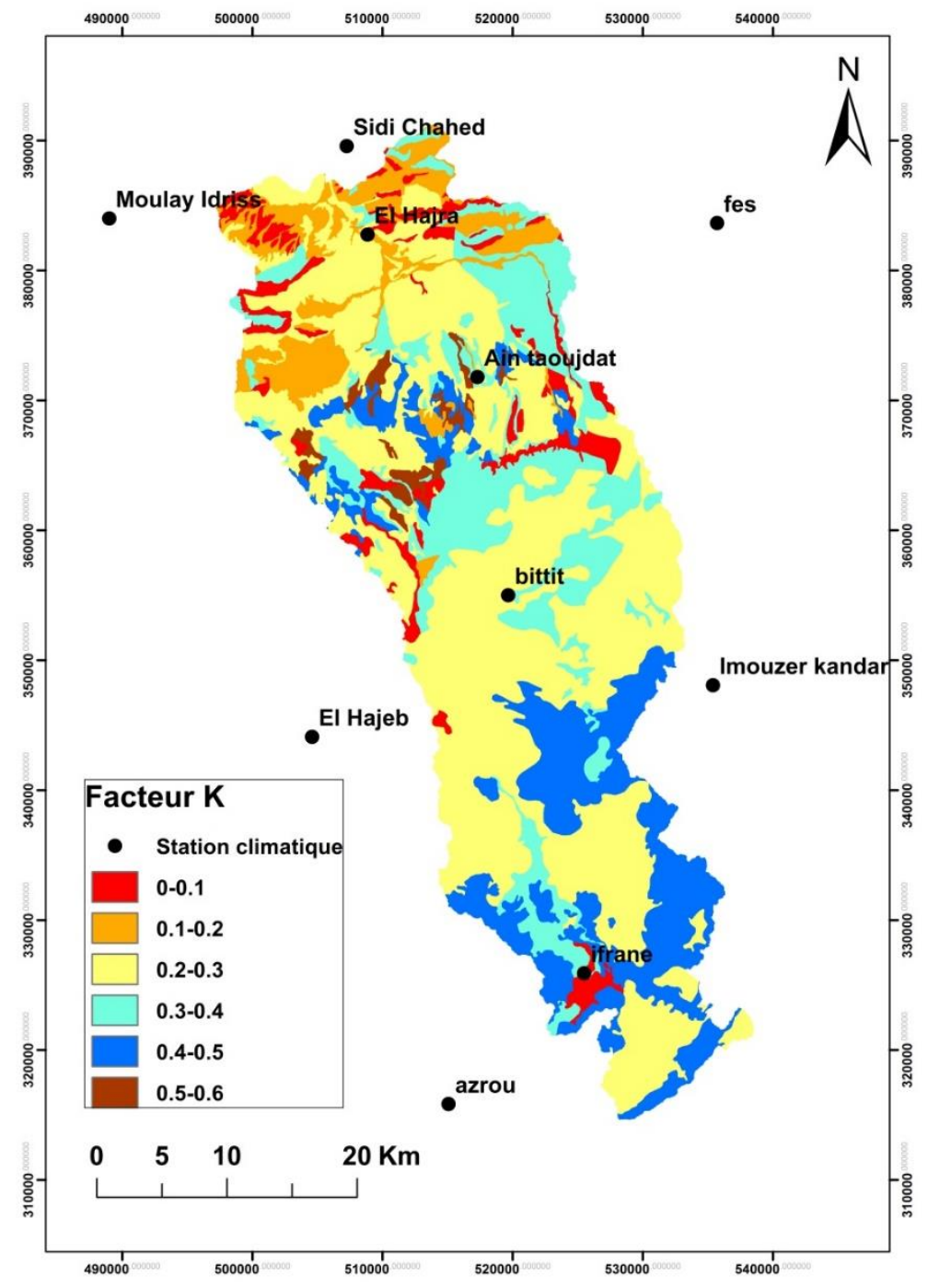

Figure 4. Carte du facteur d'érodibilité K au niveau du bassin versant de l'Oued Mikkès.

Tableau 4. Superficie et pourcentage des classes du facteur K.

\begin{tabular}{|c|c|c|}
\hline Classes de K & Superficie (ha) & $\%$ \\
\hline $0-0,1$ & 8246 & 6 \\
\hline $0,1-0,2$ & 11311 & 8 \\
\hline $0,2-0,3$ & 71394 & 49 \\
\hline $0,3-0,4$ & 25886 & 18 \\
\hline $0,4-0,5$ & 25167 & 17 \\
\hline $0,5-0,6$ & 2470 & 2 \\
\hline
\end{tabular}




\subsection{Le facteur topographique « LS »}

Le facteur LS varie selon la longueur de la pente L (m) et son degré d'inclinaison S (\%). Les résultats obtenus dans la Figure 5 montrent que les causses moyens atlasiques ainsi que les collines Pré-rifaines, qui se caractérisent par un relief fort sont les zones ou le facteur LS est le plus élevé. Le reste du bassin versant, surtout dans la plaine de Saïs, montre des valeurs modérées à faibles (Tableau 5). Le facteur LS sera donc peu influençant sur l'érosion à l'échelle du bassin versant.

Le Tableau 5 montre que $73 \%$ de la superficie du bassin versant font partie de la classe $0-5,12 \%$ sont occupés par la classe $5-10$ et $15 \%$ appartiennent à la classe supérieure à 10.

Par contre dans le bassin du haut Oum Rbiaa (Yjjou et al., 2014), la majeure partie du bassin est soumise à un grand risque d'érosion dont $62 \%$ de la superficie du bassin versant fait partie de la classe 5 à 30 .

D'après (Zingg, 1940) cité par (El Bouqdaoui, 2007), le degré d'érosion augmentait de façon exponentielle avec le degré de pente. Il avait également conclu que les pertes en sol étaient en fonction de la longueur de la pente.

Tableau 5. Superficie et pourcentage des classes du facteur LS.

\begin{tabular}{|c|c|c|}
\hline Classe du LS & Superficie (ha) & Superficie (\%) \\
\hline $0-5$ & 104145 & 73 \\
\hline $5-10$ & 17254 & 12 \\
\hline $10-15$ & 8661 & 6 \\
\hline $15-20$ & 4785 & 3 \\
\hline$>20$ & 8706 & 6 \\
\hline
\end{tabular}




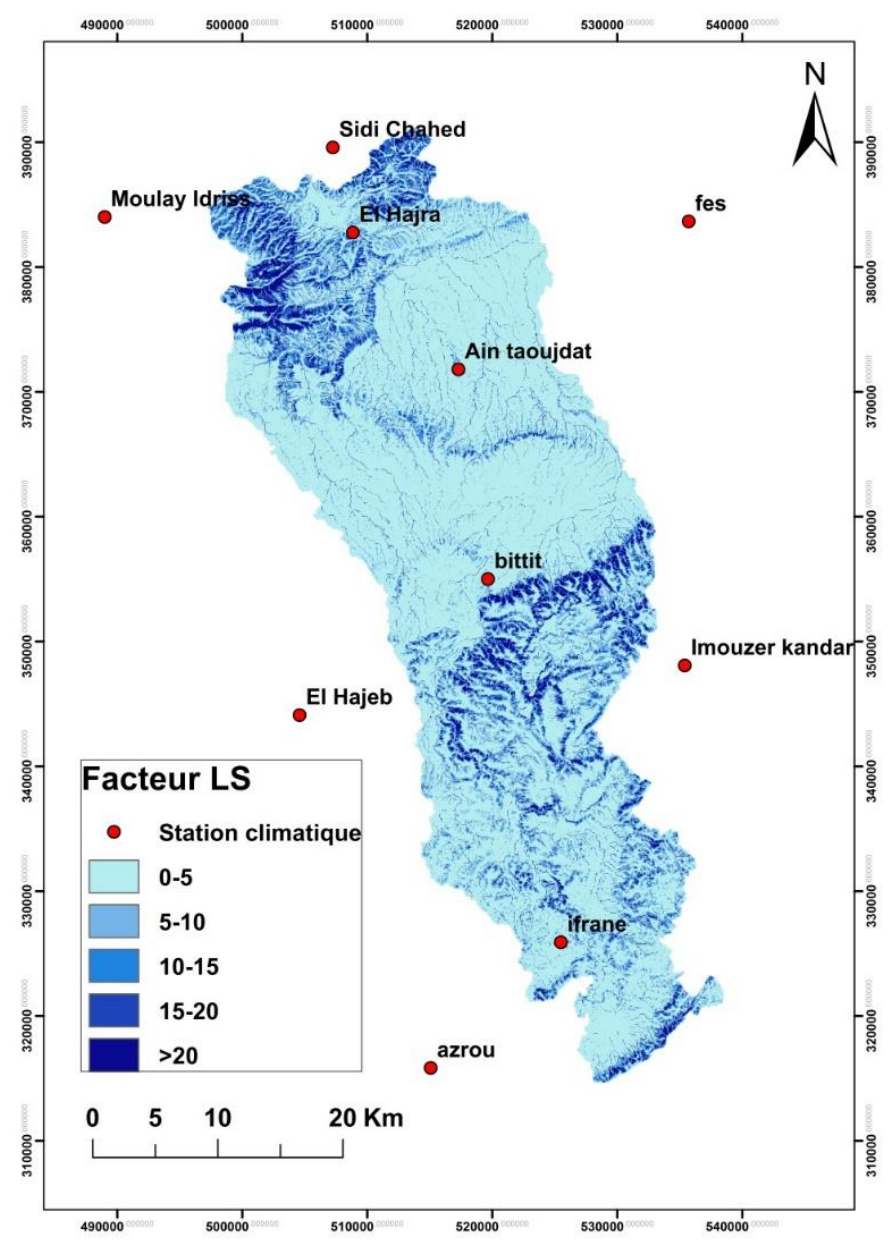

Figure 5. Carte du facteur topographique LS au niveau du bassin versant de l'Oued Mikkès.

\subsection{Le facteur du couvert végétal « $C$ »}

La carte du facteur $\mathrm{C}$ déduite à partir de la carte d'occupation du sol montre que les zones en amont du bassin versant couvertes par les forêts sont les seules qui sont relativement protégés contre l'érosion par rapport au zones médianes et avales. Les sols de cultures et des parcours situés sur les piedmonts et sur la plaine du Saïs présentent des valeurs modérées à élevées (Figure 6). Le tableau 6 montre que 90\% de la superficie du bassin représente un taux de couverture végétale très faible $(C$ supérieur à 0,4$)$ et que seulement $10 \%$ sont bien protégés avec une valeur de $\mathrm{C}$ inférieur à 0,4 (Tableau 1 ).

Ces résultats sont très proches de ceux obtenus dans l'étude réalisée sur le bassin d'Oued Beht (DREF, 2007), avec 70\% de sa superficie présente un taux de couverture faible à très faible et la protection n'est constatée que 
sur $15 \%$ du bassin. Ainsi, il est conclu que l'érosion potentielle au niveau de ce bassin est fortement accélérée.

De même, dans le bassin versant du haut Oum Er-Rbia, Yjjou et al. (2014) ont trouvé que 64\% de la superficie du bassin présente une protection très faible et que $12 \%$ seulement est bien protégée $(C<0,2)$.

Dans la même optique Goor.f (2005) a montré que le risque d'érosion hydrique est plus élevé lorsque les sols sont occupés par des cultures de type sarclé peu couvrantes ou des plantes pastorales dégradées, moins élevé avec des cultures de type non sarclé, plus couvrantes, alors que le risque est minimal quand il s'agit des prairies et des forêts denses.

Aussi, le sol peut s'influencer par les cultures qui nécessitent un affinement de la couche superficielle du sol, ce qui peut entrainer la formation d'une croûte de battance qui en cas de fortes pluies, réduit l'infiltration et accélère le ruissellement.

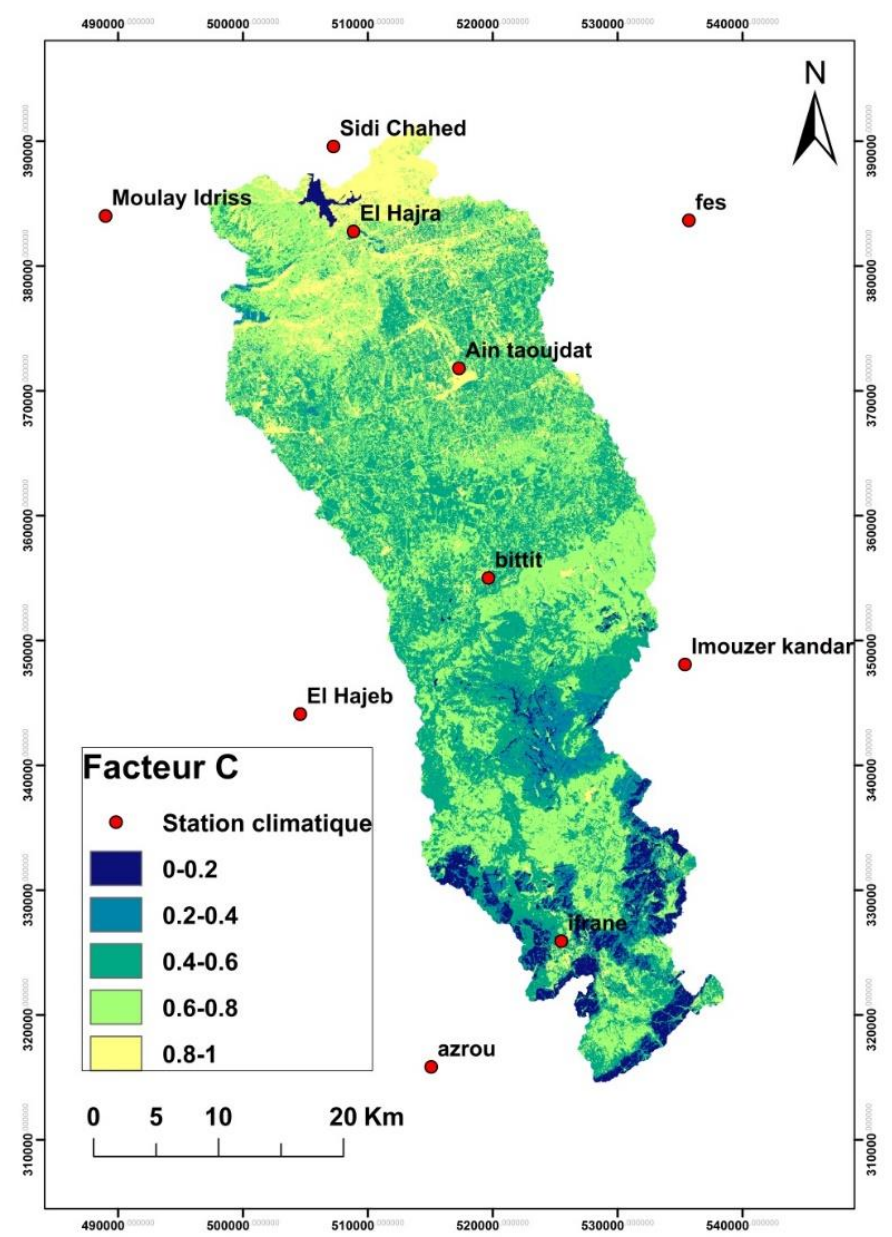

Figure 6. Carte du facteur C au niveau du bassin versant de l'Oued Mikkès. 
Tableau 6. Superficies des classes du facteur végétal C.

\begin{tabular}{|c|c|c|}
\hline Classes de C & Superficie (ha) & $\%$ \\
\hline $0-0,2$ & 7153 & 5 \\
\hline $0,2-0,4$ & 7418 & 5 \\
\hline $0,4-0,6$ & 55986 & 39 \\
\hline $0,6-0,8$ & 56375 & 39 \\
\hline $0,8-1$ & 17569 & 12 \\
\hline
\end{tabular}

\subsection{Evaluation des pertes en sol « $A$ »}

La carte des pertes en sol élaborée par la multiplication sous SIG des cartes correspondantes aux quatre facteurs décrits précédemment montre que le bassin versant de l'Oued Mikkès présente une variabilité importante en termes d'érosion des sols, avec des valeurs allant de moins de 7 tonnes à l'hectare à plus de 100 tonnes à l'hectare (Figure 7). Les zones en amont sont les plus sujettes à l'érosion en raison, notamment, du relief mouvementé et du manque de couvert végétal. Les crêtes des bordures du Moyen Atlas au Nord sous parcours dégradés, ainsi que les sommets des collines marneuses cultivées du Pré-rifs au Sud sont particulièrement vulnérables. Ces deux zones contribueraient le plus à la sédimentation au niveau du barrage en aval. La plaine du Saïs, est en général modérément sujette à l'érosion, grâce à la faible variabilité de sa topographie.

La classe des sols à pertes inférieures à la limite de $7 \mathrm{t} / \mathrm{ha} / \mathrm{an}$, considérée comme seuil de tolérance (Renard et al., 1996) occupent seulement $11 \%$ du territoire du bassin versant.

Sur le bassin versant du haut Oum Rbiaa, Yjjou et al. (2014) ont mis en évidence que les pertes sont très focalisées dans l'intervalle 50-400 t/ha/an, soit $54 \%$ du territoire. Alors que les faibles pertes en dessous du seuil de tolérance concernent 9,19\% de la superficie du bassin.

Ces résultats sont proches de ceux obtenus sur le bassin versant d'Oued Beht (DREF, 2007) qui présentent une forte érosion (entre 50 et $400 \mathrm{t} / \mathrm{ha} / \mathrm{an}$ ) sur $45 \%$ de son territoire tandis que les faibles pertes $(<7 \mathrm{t} / \mathrm{ha} / \mathrm{an})$ couvrent $12,7 \%$ seulement. 


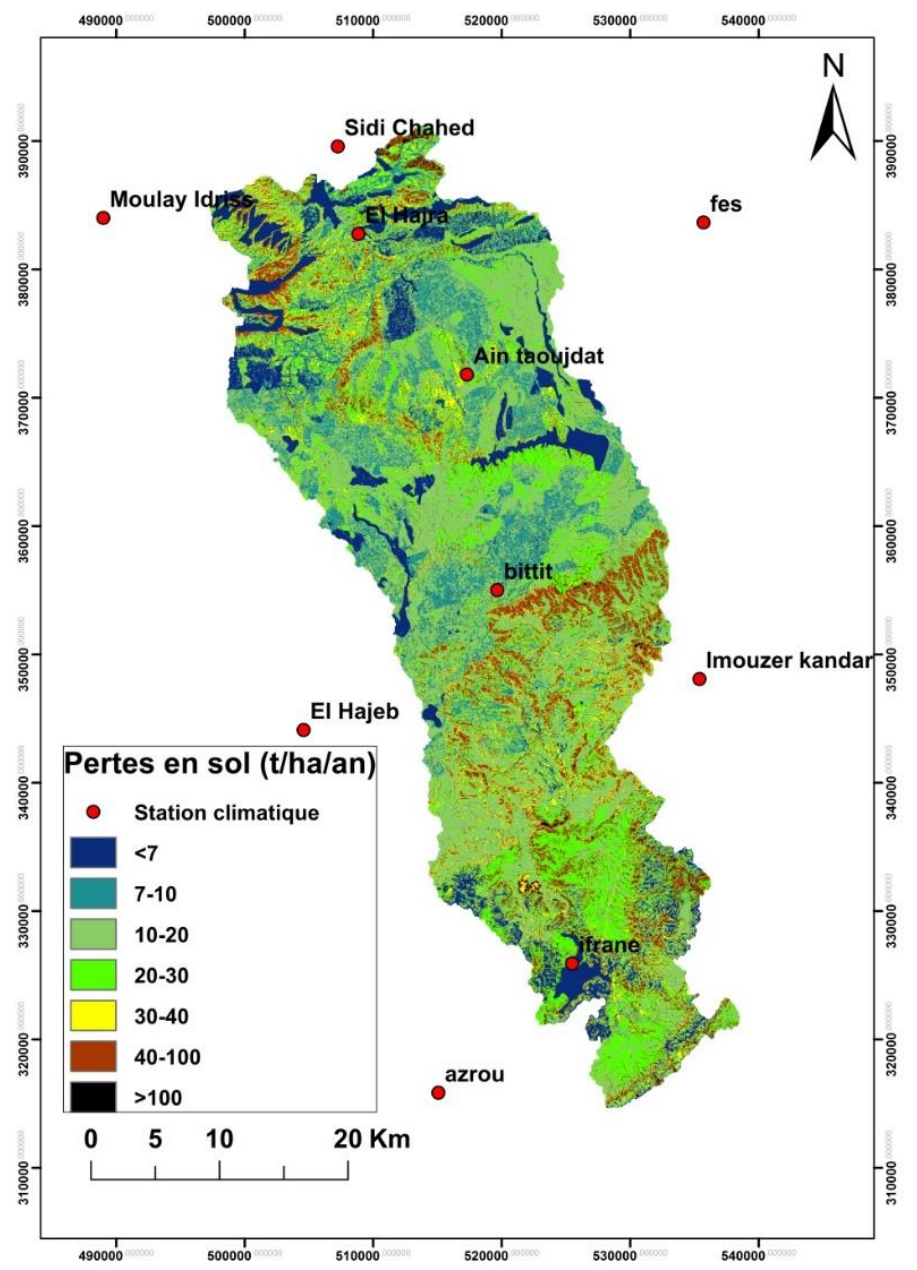

Figure 7. Carte des pertes en sol au niveau du bassin versant de l'Oued Mikkès

Les terres présentant des pertes variant de 7 à $20 \mathrm{t} / \mathrm{ha} / \mathrm{an}$ occupent $53 \%$ de la superficie du bassin, celles présentant 20 à 40 t/ha/an occupent près de $24 \%$, et celles dépassant les $40 \mathrm{t} / \mathrm{ha} / \mathrm{an}$ concernent environ $12 \%$ du territoire (Tableau 7).

Tableau 7. Superficie et pourcentage des classes des pertes en sol en t/ha/an.

\begin{tabular}{|c|c|c|}
\hline Classe des pertes en sol A & Superficie (ha) & Superficie (\%) \\
\hline$<7$ & 16063 & 11 \\
\hline $7-10$ & 16192 & 11 \\
\hline $10-20$ & 59732 & 42 \\
\hline $20-30$ & 25519 & 18 \\
\hline $30-40$ & 9217 & 6 \\
\hline $40-100$ & 16345 & 11 \\
\hline$>100$ & 1048 & 1 \\
\hline
\end{tabular}


Ainsi, considérant le seuil de tolérance à $7 \mathrm{t} / \mathrm{ha} / \mathrm{an}$, on peut constater que la majorité du bassin versant est sujette à des érosions importantes et à des degrés variables sur près de $89 \%$ du bassin versant. Les pertes ainsi engendrées représentent une menace importante à moyen et long termes de sédimentation au niveau du barrage Sidi Chahed à l'exutoire du bassin versant.

Des valeurs similaires de pertes en sols ont été également obtenues dans d'autres bassins versants du Moyen Atlas dont les caractéristiques sont proches de celles du bassin versant de Mikkès. Yjjou et al. (2014) ont rapporté des pertes en sol dans le bassin d'Oum Rbiaa au Moyen Atlas variant entre 50 et $400 \mathrm{t} / \mathrm{ha} / \mathrm{an}$, avec seulement $9 \%$ des terres en dessous du seuil de tolérance $(<7 \mathrm{t} / \mathrm{ha} / \mathrm{an})$.

Les zones les plus vulnérables à l'érosion, telles que illustrées par la figure 9, méritent ainsi une attention particulières en termes de bonnes pratiques de gestion et de conservation des sols pour atténuer les pertes en sol et éviter la dégradation de la qualité des terres et leur productivité.

Pour avoir un intérêt primordial des résultats déduits de l'application du modèle RUSLE de la zone d'étude, la comparaison des résultats avec plusieurs cas au Maroc est nécessaire pour la reconnaissance et la hiérarchisation des principales zones sources de sédiments :

- Bassin versant Tigrigra, Benamar (2009): il est situé au point de rencontre du Moyen Atlas central avec le plateau central sur une superficie de 95044 ha. L'étude de l'érosion réalisée au niveau de ce bassin, en utilisant la formule de Wischmeier révisée, a montré une érosion moyenne de 21,3 t/ha/an. La valeur maximale des pertes a atteint $32 \mathrm{t} / \mathrm{ha} / \mathrm{an}$ touchant $20,4 \%$ de la superficie du bassin.

- Bassin versant d'Oum Rbiaa, Yjjou et al. (2014) : l'étude a montré que ce bassin est soumis à une forte érosion. Ainsi, des pertes variant de 50 à 400 t/ha/an, touchent $54 \%$ du territoire. Par contre les faibles pertes en dessous du seuil de tolérance $(<7 \mathrm{t} / \mathrm{ha} / \mathrm{an})$ concernent $9 \%$ de la superficie du bassin.

- Bassin versant d'Oued Beht, DREF (2007): l'étude de ce bassin a montré que l'agressivité pluviale est très concentrée sur $86,8 \%$ de la superficie totale et que la topographie joue un faible rôle dans l'érosion. La bonne protection par le couvert végétal ne concerne que $15 \%$ du bassin. Pour l'érodibilité, la moitié des sols du bassin possèdent un facteur $\mathrm{K}$ entre 0,4 et 0,5 . Pour le facteur $\mathrm{A}$, les pertes fortes (>100t/ha/an) concernent 39,6\% du territoire.

- Bassin versant d'oued Srou, Elbouqdaoui et al. (2006) : cette étude est basée sur l'utilisation du modèle d'évaluation de l'érodibilité des terres LEAM ou Land Erodibility Assessment Methodology qui a été développé par Manrique (1988) sur la base de l'équation universelle de Wischmeier. Ce bassin versant est situé au Moyen Atlas, cette étude 
a révélé que la superficie vulnérable au risque d'érosion élevé, très élevé et extrêmement élevé, représente $28 \%$ de la superficie totale du bassin en année sèche. Alors que pour les mêmes classes de risque, en période humide, cette superficie représente $43 \%$. Ce qui témoigne selon les auteurs l'influence des précipitations sur les phénomènes érosifs.

- Bassin versant Nakhla, Brahim et al. (2003): d'une superficie d'environ $110 \mathrm{~km} 2$, ce bassin versant est situé dans le Rif Occidental au Nord du Maroc. Cette étude est basée sur l'utilisation de l'équation universelle révisée des pertes en sols. Les résultats obtenus montrent que le taux d'érosion est élevé au niveau des zones à pentes fortes oscillants entre 12 et 40 degrés. En effet, ces zones à prédominance de sols calcimagnésiques subissent des pertes de l'ordre de 95,5 t/ha/an.

\section{Conclusion}

Le présent travail est réalisé dans le but de quantifier les pertes en sol au niveau du bassin versant de l'Oued Mikkès en utilisant l'Equation Universelle des Pertes en Sol révisée (RUSLE) et l'outil SIG. Le bassin versant étudié constitue une liaison entre le Moyen Atlas, la plaine de Saïs et le Pré-rif. Ses altitudes varient entre $2091 \mathrm{~m}$ à $184 \mathrm{~m}$ et ses pentes entre 0 et $189 \%$. Il se caractérise par une érosion importante, avec $89 \%$ de la superficie du bassin dépassent le seuil de tolérance et $11 \%$ seulement des pertes sont inférieurs à $7 \mathrm{t} / \mathrm{ha} / \mathrm{an}$. Ceci est en relation avec les différentes valeurs des facteurs impliqués dans l'érosion hydrique des sols. Les valeurs du facteur d'érosivité R sont très élevées ; en effet, $99 \%$ de la superficie du bassin présente des valeurs qui varient entre 60 et 138, avec un gradient de l'amont vers l'aval. De même, le facteur d'érodibilité K présente des valeurs élevées sur $86 \%$ de la superficie globale. Le facteur topographique LS est plus influençant sur les montagnes du Moyen Atlas et des rides Pré-rifaines, et sans effets majeur sur la plaine du Sais. Le bassin versant de l'Oued Mikkès se caractérise par un taux de couverture végétale très faible avec $90 \%$ de la superficie ayant une valeur de $\mathrm{C}>0,4$ une dominance des terrains de parcours, des terrains incultes et des terrains de cultures qui sont considérés sensibles à l'érosion. De manière général, le bassin versant de l'Oued Mikkès est exposé à un grand risque d'érosion hydrique. Cette érosion a des retombées négatives sur la qualité des sols dans presque tout le bassin versant (89\% de la superficie du bassin versant), en détruisant la structure, la texture et la qualité des sols, ainsi que sur le transport des sédiments et l'envasement du barrage en aval d'un taux de 0.3Mm3/an (Ministère de l'Aménagement du Territoire, de l'Eau et de l'Environnement).

Les résultats obtenus donnent une idée importante pour que les décideurs connaissent les zones à risques et proposent les interventions 
adéquates pour la lutte contre l'érosion à partir de la description approfondie de chacun des facteurs liés aux processus d'érosion. Il existe certaines recommandations à suivre pour lutter contre le risque d'érosion telles que par exemple ajouter à l'équation universelle des pertes en sol de Wischmeier d'autres modèles pour calculer la quantité de sédimentation produite au niveau du bassin versant étudié ainsi que le taux du phosphore et de la matière organique, et tenir compte de l'importance relative des pressions agricoles et non agricoles et de la fragilité du milieu et programmer des interventions pour stabiliser le sol et améliorer l'infiltration des eaux et diminuer l'impact topographique.

\section{References :}

1. Ait Fora, A., 1995. Modélisation spatiale de l'érosion hydrique dans un bassin versant du roc marocain: validation de l'approche géomatique par la sédimentologie, les traceurs radio-actifs et la susceptibilité magnétique des sédiments.

2. Brahim, L.A., Alaoui, F.S., Siteri, H., Tahri, M., 2003. Quantification des pertes en sols dans le bassin-versant Nakhla (Rif septentrional). Science et changements planétaires/Sécheresse 14, 101-106.

3. DREF, du N.-E., 2007. Etude d'aménagement du bassin versant d'Oued Beht en amont du barrage El Kansera.

4. El Bouqdaoui, K., 2007. Approche méthodologique de l'évaluation du risque potentiel d'érosion des sols du bassin versant du Srou (Moyen Atlas, Maroc) à l'aide de la télédétection et du SIG.

5. EL Idrissi.R, 1986. Etude des sols en vue de la mise en valeur agricoleProvince de Khénifra. Projet de développement agricole intégré. Ministère de l'Agriculture et de la Réforme Agraire, Institut National de la Recherche Agronomique, D. P. A., Khénifra, Maroc. Rapport et cartes.

6. Elbouqdaoui, K., Ezzine, H., Zahraoui, M., Rouchdi, M., Badraoui, M., 2006. Évaluation du risque potentiel d'érosion dans le bassinversant de l'oued Srou (Moyen Atlas, Maroc). Science et changements planétaires/Sécheresse 17, 425-431.

7. Goor.f, 2005. L'érosion hydrique des sols dans la région wallonne (année 2005) chapitre 11 les sols et l'environnement terrestre, http://environnement.wallonie.be.

8. Lahlou, A., 1977. Dégradation spécifique des bassins versants au Maroc. Rabat: Rapport $\mathrm{n}^{\circ} 1000$.Ministère de l'équipement et de la promotion nationale, Direction de l'hydraulique, Division exploitation, service de gestion des eaux ; $161 \mathrm{p}$.

9. Lufafa, A., Tenywa, M., Isabirye, M., Majaliwa, M., Woomer, P., 2003. Prediction of soil erosion in a Lake Victoria basin catchment 
using a GIS-based Universal Soil Loss model. Agricultural systems 76, 883-894.

10. Manrique, L.A., 1988. Land erodibility assessment methodology (LEAM): using soil survey data based on soil taxonomy. Editorial and Publication Shop.

11. Marzouki, T., 1992. Diagnostic de l'envasement des grands barrages au Maroc. Revue marocaine du génie civil 38: 46-50.

12. Moore, I.D., Wilson, J.P., 1992. Length-slope factors for the Revised Universal Soil Loss Equation: Simplified method of estimation. Journal of soil and water conservation 47, 423-428.

13. Rango, A., Arnoldus, H.M.J., 1987. Aménagement des bassins versants. Cahiers techniques de la FAO 1-11.

14. Renard, K.G., Foster, G.R., Wessies, G.A., Mc Cool, D.K., Yoder, D.C., 1996. Predicting soil erosion by water: a guide to conservation planning with the Revised Universal Soil Loss Equation (RUSLE). US Dept. Agric. Handbook 703, 384 p. US Dept. Agric. Handbook 703, $384 \mathrm{p}$.

15. Wischmeier, Smith, D.D., 1978. Predicting rainfall erosion losses-a guide to conservation planning. Predicting rainfall erosion losses-a guide to conservation planning.

16. Wischmeier, W.H., Smith, D.D., 1978. Predicting rainfall erosion losses-a guide to conservation planning. Predicting rainfall erosion losses-a guide to conservation planning.

17. Yjjou, M., Bouabid, R., El Hmaidi, A., Essahlaoui, A., El Abassi, M., 2014. Modélisation de l'érosion hydrique via les SIG et l'équation universelle des pertes en sol au niveau du bassin versant de l'Oum ErRbia. The International Journal Of Engineering And Science (IJES) 3, 83-91.

18. Zingg, A.W., 1940. Degree and length of land slope as it affects soil loss in run-off. Agric. Engng. 21, 59-64.

19. Zouagui, A., Sabir, P.M., Naimi, P.M., Chikhaoui, P.M., Benmansour, M., 1977. Modelisation Du Risque D'érosion Hydrique Par L'équation Universelle Des Pertes En Terre Dans Le Rif Occidental: Cas Du Bassin Versant De Moulay Bouchta (Maroc). European Scientific Journal January 2018 edition Vol.14, No.3 ISSN: 1857 - 7881 (Print) e - ISSN 1857- 7431. 